\title{
Protocol
}

\section{Effectiveness of a 12-week physical exercise programme on blood pressure in adults with true resistant hypertension and high risk of obstructive sleep apnoea: a study protocol for a pre-post test non randomised clinical trial}

\author{
Suranga Dassanayake ${ }^{1}$, Gerard Wilkins ${ }^{2}$, Gisela Sole ${ }^{1}$, Margot Skinner ${ }^{1 *}$ \\ ${ }^{1}$ Centre for Health, Activity and Rehabilitation Research, School of Physiotherapy, University of Otago, New Zealand \\ ${ }^{2}$ Department of Medicine, Dunedin School of Medicine, University of Otago, New Zealand
}

Received: 21 January 2020

Revised: 19 March 2020

Accepted: 31 March 2020

\section{*Correspondence:}

Dr. Margot Skinner,

E-mail: margot.skinner@otago.ac.nz

Copyright: (C) the author(s), publisher and licensee Medip Academy. This is an open-access article distributed under the terms of the Creative Commons Attribution Non-Commercial License, which permits unrestricted non-commercial use, distribution, and reproduction in any medium, provided the original work is properly cited.

\section{ABSTRACT}

Background: Resistant hypertension, a special phenotype of hypertension, is associated with increased cardiovascular risk. Exercise and physical activity are recommended as non-pharmacological interventions to manage blood pressure in hypertension. Little is known about the effectiveness of exercise in resistant hypertension. A bidirectional relationship has been identified between resistant hypertension and obstructive sleep apnoea but the literature pertaining to the benefit of exercise for populations with both conditions, is minimal. This study aims to identify the effectiveness of exercise in reducing blood pressure in a cohort of adults with resistant hypertension and high risk of obstructive sleep apnoea.

Methods: Dunedin based adults with resistant hypertension and high risk of obstructive sleep apnoea will be recruited from the community and health centres to participate in the study. Consenting volunteers (no. of fourteen) will participate in a 12-week exercise programme including aerobic and strength training. The primary outcome measure will be $24 \mathrm{~h}$ ambulatory blood pressure while the secondary outcomes will be anthropometrics, activity parameters, sleep parameters, cardiac structure and function, and quality of life. The measurements at the end of the 12-week exercise programme will be compared with baseline to determine the effectiveness of exercise in reducing blood pressure in the cohort with resistant hypertension and risk of obstructive sleep apnoea.

Conclusions: Exercise and physical activity are recommended to manage hypertension. Therefore, it is anticipated that the exercise programme will have a positive effect on the blood pressure of the participants. The findings have potential to change the direction of research in the area by filling the knowledge gaps.

Trial Registration: The Ministry of Health, New Zealand (Ethics Ref: 18/CEN/257), Trial registry: ACTRN12618001881224p).

Keywords: Resistant hypertension, Obstructive sleep apnoea, Exercise, Study protocol, Non-randomised trial

\section{INTRODUCTION}

Hypertension is a worldwide problem, which affects billions of people. ${ }^{1}$ The global prevalence of hypertension in adults is over $25 \%$ and the New Zealand prevalence is over $30 \%$ with ethnic disparities., ${ }^{2,3}$ Māori have the highest risk as well as having a $15 \%$ higher use of antihypertensive medications compared to New Zealand Europeans. ${ }^{3}$ Cardiovascular disease (CVD) is the greatest cause of morbidity and mortality in the New Zealand population and hypertension increases the CVD risk. ${ }^{2,3}$ The new American College of Cardiology or American 
Heart Association (ACC or AHA) guideline, defines stage 1 hypertension as 130 to $139 \mathrm{mmHg}$ systolic or 80 to $89 \mathrm{mmHg}$ diastolic blood pressure (BP), and Stage 2 hypertension as $140 / 90 \mathrm{mmHg}$ or higher., ${ }^{4,5}$ The New Zealand Guideline (2018) has been updated to match this. $^{2}$

Both the level and range of physical activities undertaken by individuals are potential predictors of hypertension. ${ }^{6}$ High-grade evidence has shown that exercise, especially aerobic exercise combined with resistance training, lowers high blood pressure as well as having other therapeutic effects including lowering blood cholesterol and weight reduction. ${ }^{6-8}$ Importantly, there is consensus within the international guideline groups - AHA or ACC UK, European Society of Hypertension and European Society of Cardiology (ESH and ESC) - that the management of hypertension in the lower range (stage I or pre-hypertension), focusses on lifestyle factors in particular, physical activity, and exercise rather than pharmacological interventions. ${ }^{4,7,9-11}$

Resistant hypertension was previously considered to be a rare and diminishing phenomenon but in recent years it has been recognised as an increasingly common and alarming health problem, which increases CVD risk, mortality and morbidity. ${ }^{1,12,13}$ Resistant hypertension is defined as, 'blood pressure that remains above the goal in spite of the concurrent use of three antihypertensive agents of different classes including a diuretic or use of four or more medications regardless of BP values,' (AHA Scientific Statement of 2008, page e511 and 2018, page e54). ${ }^{12,13}$ Noubiap et al have confirmed that the global prevalence of true resistant hypertension is $10.3 \%(95 \%$ CI $7.6 \%$ and $13.2 \%$ ) among the population with hypertension. ${ }^{14}$

Ambulatory BP taken over $24 \mathrm{~h}$ has been shown to be the most effective tool to validate resistant hypertension as being true resistant hypertension. ${ }^{5}$ However, a key limitation of many studies that have investigated the management of resistant hypertension by focusing on lifestyle changes, such as an increase in physical activity, have not used $24 \mathrm{~h}$ ambulatory BP to diagnose true resistant hypertension within their cohorts. ${ }^{15-17}$

Studies have shown that aerobic and resistance exercise together, have a positive effect on resistant hypertension because the exercise will reduce BP directly through changes in predisposing factors, including increased arterial stiffness and sympathetic tone, and by reducing obesity. ${ }^{18}$ High-level research evidence from recent randomised control trials and meta-analyses supports a clinically significant reduction in ambulatory systolic BP of 4-9 $\mathrm{mmHg}$ as a result of programmed exercise and physical activity. ${ }^{15,19,20}$ The literature confirms that there is a $20 \%$ and $7 \%$ CVD risk reduction in adults with hypertension by dropping $\mathrm{BP}$ by $10 \mathrm{mmHg}$ and a $7 \%$ CVD risk reduction with a $5 \mathrm{mmHg}$ drop. ${ }^{21}$ Thus it can be surmised that in the cohort of adults with resistant hypertension, undertaking a prescribed physical activity program may lead to a reduction in BP, and thereby, a reduction in CVD risk as well as in the number of antihypertensive medications required..$^{5,12}$

Obstructive sleep apnea (OSA) in adults has been correlated with the degree of BP elevation above normal and thereby with an increased risk of hypertension. ${ }^{22}$ OSA has a close and strong relationship with resistant hypertension and is cited as the most common secondary cause. $^{23,24}$ It follows that since the severity of OSA is associated with major modifiable risk factors, such as obesity and physical inactivity, in patients with resistant hypertension, reducing OSA through modifying the risk factor/s may be highly beneficial. ${ }^{25}$ The widely used treatment for managing the obstruction in OSA is continuous positive airway pressure (CPAP) ${ }^{26}$ However, the CPAP may be identified as a symptomatic treatment modality rather than a curative modality. ${ }^{27}$ Rather than just focusing on symptomatic management of OSA, it is suggested that a therapeutic strategy such as exercise, is a more effective modality to address OSA and resistant hypertension simultaneously. ${ }^{25}$ It has also been shown, that a prescribed physical activity and exercise program can lead to a reduction in BP in individuals with high risk of OSA and hypertension. ${ }^{28,29}$

In summary, physical inactivity is considered to be a modifiable risk factor, for the two conditions - resistant hypertension and OSA. There is a high level of evidence for the therapeutic effects of exercise on hypertension and OSA as well. However, published research on the effectiveness of exercise on the cohort of adults with resistant hypertension and high risk of OSA is very limited, and further investigations are warranted. We thus developed the study protocol based on the hypothesis that a 12-week physical exercise program will provide a clinically significant change in $24 \mathrm{~h}$ ambulatory BP, in a cohort of adults with true resistant hypertension and OSA; and activity levels, cardiovascular fitness, structure and function of the heart, sleep and anthropometry parameters will also improve.

\section{Aim of the study}

The main aim of this study is to investigate the effectiveness of a 12-week aerobic and strengthening exercise program on $24 \mathrm{~h}$ ambulatory BP in Dunedin based adults, 60 years or younger with true resistant hypertension and high risk of OSA.

\section{Specific objectives}

The specific objectives are to investigate the changes in the magnitude of $24 \mathrm{~h}$ ambulatory and clinic or office BP, activity level, sleep parameters or scores, anthropometrics, quality of life, and in structural and functional features of the heart, in response to a 12 week supervised and home based exercise program. 


\section{METHODS}

This study is the second of two studies comprising research for a $\mathrm{PhD}$. The participants eligible for this study will be identified by the research team, from amongst the participants in study I. ${ }^{1}$ Eligible participants will be invited to enter study II, the present study.

\section{Study design}

The study will be a single group pre-post test nonrandomised clinical trial.

\section{Study population and study setting}

The population for the study will be the Dunedin based adults aged 60 years and younger, diagnosed with both true resistant hypertension and a high risk of OSA.
The study will be conducted in the School of Physiotherapy, University of Otago and the echo cardiograms will be carried out in the Dunedin School of Medicine Cardiology Research Laboratory.

\section{Participant recruitment}

The initial recruitment includes adults based in the Dunedin region, aged 60 years and younger, diagnosed with high BP, who are on three or more pharmacological agents of different classes including a diuretic (AHA definition 2008 or 2018). ${ }^{12,13}$ The individuals with sleep scores and/or sleep fragmentations indicative of a high risk of OSA, will be identified by the research team. Those who are meeting the inclusion criteria outlined below, will be invited into study II. Written informed consent will be obtained from those who agree, prior to their entry into study II, the 12-week supervised exercise program (Figure 1).

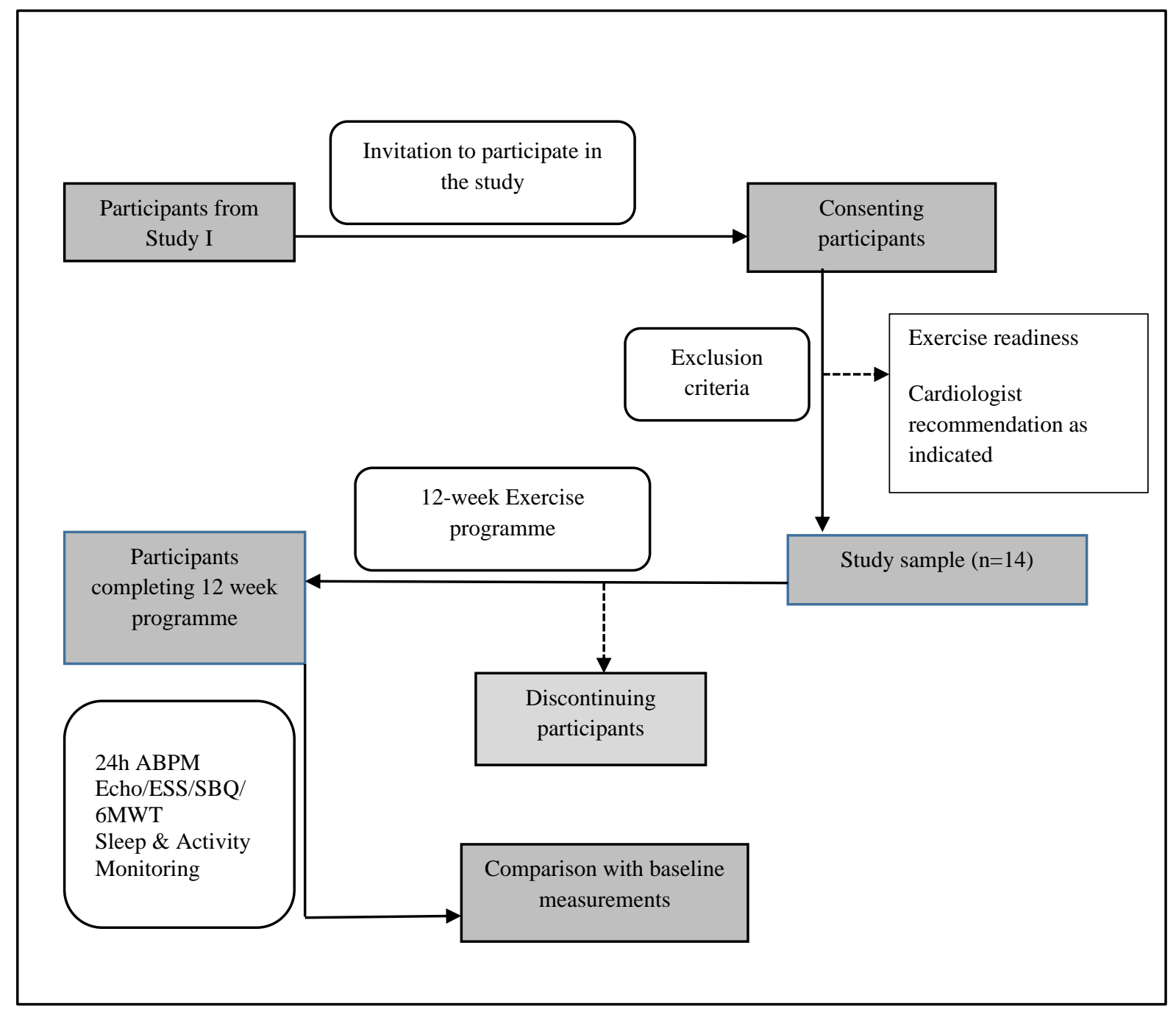

Figure 1: Flow chart of participant recruitment.

ESS: Epworth sleepiness scale, 6MWT: 6 minute walk test, TRHT: true resistant hypertension, ABPM: ambulatory blood pressure monitoring, SBQ: STOP-BANG questionnaire 


\section{Inclusion}

Adults (18-60 years inclusive), diagnosed with true resistant hypertension, and a high risk of OSA - (Epworth sleepiness scale (ESS) score $\geq 9$ and/or STOP-BANG questionnaire (SBQ) $\geq 5$ or 'high'), and cleared as being fit to participate in the 12-week physical exercise program, according to criteria from the adult pre-exercise screening tool, Exercise and Sports Science Australia (ESSA) or Fitness Australia and Sports Medicine Australia (SMA)), will be included in the study. ${ }^{30-32}$

\section{Exclusion}

Eligible participants with 'multiple risk factors' according to the adult pre-exercise screening tool e.g. blood pressure $>200 / 100 \mathrm{mmHg}$; abnormalities with echocardiograms determined as 'high risk of harm whilst participating in moderate activity' by the health professionals on the research team; will be excluded from the study. ${ }^{20,32}$ Further, the individuals with systemic diseases, history of CVDs such as myocardial infarction/stroke, within six months of the study, other severe heart conditions and with physical ailments such as acute musculoskeletal injuries, which limit their ability to carry out an exercise program, will also be excluded.

\section{Sample size}

The calculation of sample size was based on the study's primary outcome, $24 \mathrm{~h}$ ambulatory BP. The sample size was calculated to detect a clinically significant difference in ambulatory BP or office BP, the primary outcome, calculated as being $5 \mathrm{mmHg} .{ }^{15,33}$ The cohort with resistant hypertension is known for the Dunedin based population $(n=559) .{ }^{34}$ Thus, to provide $80 \%$ power and significance level at $0.05(5 \%)$ to detect an estimated difference of $5 \mathrm{mmHg}$ with standard deviation $\pm 6 \mathrm{mmHg}$, 14 participants will be needed. Therefore, the target participant number is set at 30 allowing for at least a $50 \%$ dropout rate to fulfill the sample size of 14 .

\section{Randomization}

This study will be a single group pre-post test non randomized study, which will not involve a control group.

\section{Intervention: the exercise program}

The recruitment and intervention will be carried out as described below. The reporting of the intervention has been structured in line with the template for intervention description and replication (TIDieR) guide. ${ }^{35}$ The participants will be asked not change their usual routines and other medical intervention while they are involved in the intervention. Participants will be requested to inform the researchers of any changes in their medications, health status or any other external interventions that could interfere during the period of participation. The intervention, the exercise program (Table 2) has been designed by a subject expert physiotherapist and targets the activity guidelines set by Ministry of Health New Zealand and American College of Sports Medicine. ${ }^{36,37}$ The exercise program will be discontinued on request or in the event of an adverse cardio vascular event or acute injury, as determined by regular reporting to the research supervisory team (MS/GW/or GS). The exercise program and all the measurements except the echocardiogram, will be carried out at School of Physiotherapy. The echocardiogram will be carried out in the Dunedin School of Medicine Cardiology Research Laboratory.

\section{Step I - initial recruitment}

The primary supervisor for the project (MS) will contact the participants, who will have been identified as having true resistant hypertension and high risk of OSA, from the list of the study participants in study I. Eligible and consenting participants will be assessed by the principal investigator (SD) and primary supervisor, at a face to face interview, and screened for the eligibility for exercise using the pre exercise screening test ESSA. ${ }^{32}$ In the case of a person being assessed as 'potentially high risk', further advice will be obtained by the interventional cardiologist $(\mathrm{GW})$ member of the research team, prior to the person commencing the exercise. Study participants will then be provided with an appointment to join the exercise program with a schedule (dates and time), which will enable them to participate for 12 consecutive weeks. Both the supervised and home based exercise program will be individually designed for each participant, to ensure the program is participant-centered.

\section{Step II - baseline measurements}

The parameters (Table 1) which were measured in the Study I, will be taken as the baseline measurements for the present study, study II. Participants will be recruited to study II within four weeks of their participation in study I. The $24 \mathrm{~h}$ ambulatory BP/office BP, ESS and SBQ scores may be repeated if this duration is exceeded or the treatment regimen for blood pressure has been changed after the initial measurements.

\section{Step III - intervention: the exercise program}

The intervention has been designed by an experienced research team, comprising three physiotherapists with experience and qualifications in sports science, musculoskeletal physiotherapy and cardiorespiratory physiotherapy, and an interventional cardiologist. The strengthening and aerobic exercise program will be designed based on the individual's cardiorespiratory endurance parameters such as frequency, intensity, time and type (FITT) and muscle strength parameters like repetition maximum (RM). The recommended level of exercise (Eating and Activity Guidelines for New Zealand Adults, Ministry of Health) and exercise intensity guidelines of adults pre-exercise screening tool, 
ESSA and Sports Medicine Australia, will also be targeted in the program. ${ }^{2,32,36}$ Thus, each participant will receive at least 60 minutes of exercise $(5 \mathrm{~min}$ warming up, 20-30 min aerobic training, 20-30 min resistance training and 5 min cooling down) (Table 2), along with an individualised home base program (Table 2).

The supervised exercise programme will be run by an experienced physiotherapist on a weekly basis for the period of time required for each participant to attend for 12 weeks. Basic cardiorespiratory assessments will be carried out on each participant prior to and at the end of each session. If the pre exercise blood pressure is above $200 / 100 \mathrm{mmHg}$, indicating severe hypertension, the participant will be advised not to participate in the exercise programme and directed to their general practitioner by MS or GW. ${ }^{11,20}$ Additionally the participants will be educated on the importance of regular aerobic and resistance exercise for their health, by the physiotherapist as part of the information provided in the programme.

Table 1: Measurements, measuring equipment and tools used in study I for the same cohort with true resistant hypertension and high risk of OSA.

\begin{tabular}{|c|c|}
\hline Measurements & Equipment or tool \\
\hline 24 hours ABPM* & $\begin{array}{l}\text { ABPM (90207, Spacelabs, Snoqualmie, Washington, } \\
\text { USA }\end{array}$ \\
\hline Office blood pressure* & $\begin{array}{l}\text { Digital BP monitor (Omron automatic digital blood } \\
\text { pressure monitor, HEM 7322, Netherlands) }\end{array}$ \\
\hline Herat rate and peripheral oxygen saturation & $\begin{array}{l}\text { NT1A Pulse oximeter, New Tech, Hamburg, } \\
\text { Germany }\end{array}$ \\
\hline Height & Stadiometer $(\mathrm{cm})$ \\
\hline Weight & Electronic weighing scale $(\mathrm{kg})$ \\
\hline Neck, hip, waist circumference** & Measuring tape $(\mathrm{cm})$ \\
\hline Risk of OSA $* *$ & ESS score STOP_BANG updated questionnaire \\
\hline Quality of life** & SF-36V2 \\
\hline Exercise readiness & Adult pre-exercise screening tool, Australia \\
\hline Cardiorespiratory fitness $* *$ & $6 \mathrm{MWT} /$ Borg rating of perceived exertion scale \\
\hline $\begin{array}{l}\text { Level of activity** (step count, time in } \\
\text { low/moderate/vigorous physical activity, sedentary time) }\end{array}$ & ActiGraph GT3XPB, Pensacola, Florida US. \\
\hline $\begin{array}{l}\text { Objective sleep measurements** (total sleep time, } \\
\text { awakenings, average awakenings, sleep efficiency, sleep } \\
\text { onset, latency and wake after sleep onset) }\end{array}$ & ActiGraph GT3XPB, Pensacola, Florida US. \\
\hline \multicolumn{2}{|c|}{$\begin{array}{l}\text { Echocardiography** will be carried out using the Cardiac ultrasound machine available in the laboratory, Dunedin School of } \\
\text { Medicine. }\end{array}$} \\
\hline
\end{tabular}

Table 2: Exercise programs for the cohort of participants with true resistant hypertension and high risk of OSA.

\begin{tabular}{|c|c|c|}
\hline & Supervised program & Home based program \\
\hline Setting & $\begin{array}{l}\text { School of Physiotherapy Rehabilitation } \\
\text { Laboratory }\end{array}$ & Home or any other place \\
\hline $\begin{array}{l}\text { Screening of } \\
\text { participant }\end{array}$ & $\begin{array}{l}\text { Initial screening by MS \& SD and subsequent } \\
\text { screenings by the physiotherapist conducting the } \\
\text { session } \\
\text { Tool: ESSA }\end{array}$ & \\
\hline Equipment & $\begin{array}{l}\text { Cycle ergometers or armogometers or rowing } \\
\text { ergometers } \\
\text { Dumbbells or barbells or elastic resistance bands } \\
\text { or PRE (quadriceps) benches } \\
\text { medicine balls or gym balls } \\
\text { steps or benches }\end{array}$ & $\begin{array}{l}\text { Bicycles or static bicycle or tread mill } \\
\text { Dumbbells or Elastic resistance bands } \\
\text { Medicine balls or gym balls } \\
\text { Steps or benches }\end{array}$ \\
\hline $\begin{array}{l}\text { Duration } \\
\text { (time) } \\
45-60 \text { minutes }\end{array}$ & $\begin{array}{l}\text { Warm up- } 5 \text { min } \\
\text { Aerobic training- } 20-30 \mathrm{~min} \\
\text { Strength training- } 10-20 \mathrm{~min} \\
\text { Stretching and warm down- } 5 \mathrm{~min}\end{array}$ & $\begin{array}{l}\text { Warm up- } 5 \text { min } \\
\text { Aerobic exercise up to } 30 \mathrm{~min} \\
\text { Resistant exercise- } 10-20 \mathrm{~min} \\
\text { Stretching and warm down- } 5 \mathrm{~min}\end{array}$ \\
\hline
\end{tabular}




\begin{tabular}{|c|c|c|}
\hline & Supervised program & Home based program \\
\hline Frequency & Once a week for 12 weeks & 3-5 days a week for 12 weeks \\
\hline Intensity & $\begin{array}{l}\text { Moderate }(60-80 \% \text { HRR or } 3-5 \text { Borg RPE } \\
\text { [modified] scale }\end{array}$ & $\begin{array}{l}\text { Moderate intensity 3-5 Borg RPE [modified] } \\
\text { scale }\end{array}$ \\
\hline Type & $\begin{array}{l}\text { Aerobic warm-up } \\
\text { Aerobic training- cycling or rowing } \\
\text { Strength training- free weights or elastic bands } \\
\text { Cooling down stretching and breathing }\end{array}$ & $\begin{array}{l}\text { Warm-up } \\
\text { Aerobic training- cycling or walking } \\
\text { Strength training- free weights or elastic bands } \\
\text { Cooling down- self stretching and relaxed } \\
\text { breathing }\end{array}$ \\
\hline Exercises & $\begin{array}{l}\text { Warm up: Aerobic full body movements to a } \\
\text { rhythms of music designed for aerobic warming } \\
\text { up ( } 5 \text { min) } \\
\text { Aerobic training (AT): ( } 20 \text { min) Cycling and or or } \\
\text { rowing } \\
\text { Progression AT: resistance or time of cycling and } \\
\text { rowing (Based on HRR or RPE), speed and the } \\
\text { distance covered per unit time } \\
\text { Resistance training (RT): ( } 20 \text { min) } \\
\text { Resisted movement (Flexion, extension, medial } \\
\text { and lateral rotations, abduction, adduction) of } \\
\text { major joints (shoulder, elbow, wrist, hip, knee } \\
\text { ankle). Major muscle groups (e.g. Flexors, } \\
\text { abductors) will be targeted using body weight } \\
\text { (Squats or lunges or push ups), free weights } \\
\text { (dumbbells, barbells), resistant bands and the } \\
\text { progressive exercise equipment (Quadriceps } \\
\text { benches, total gym etc.) } \\
\text { Number of exercises: Up to } 10 \text { Maximum number } \\
\text { of repetition } 10 \times 3 \text { sets Progression RT: Repetition } \\
\text { or load } \\
\text { Cooling down: ( } 5 \text { min) } \\
\text { Slow activities (marching on the spot); stretching } \\
\text { of all major muscle groups and relaxed breathing }\end{array}$ & $\begin{array}{l}\text { Warm up: walking or cycling at acceptable speed } \\
\text { ( } 5 \text { min) } \\
\text { Aerobic exercise: } \\
\text { Activity: Brisk walking }(2-3 \mathrm{~km}) \text { or jogging (1.5- } \\
2 \mathrm{~km}) \text { or cycling ( } 3-6 \mathrm{~km}) \\
\text { Duration: } 20-30 \text { minutes, } \\
\text { Intensity: Up to exertion level } 3-5 \text { on the Borg's } \\
\text { RPE [modified] scale } \\
\text { Progression: By increasing the distance or } \\
\text { reducing duration } \\
\text { Resistance training (RT): ( } 20 \text { min) } \\
\text { Exercises } \\
\text { Seated- lateral arm raise; Seated - shoulder press; } \\
\text { Seated- overhead triceps extension; Seated - front } \\
\text { arm raise; Seated - biceps curl - with dumbbells: } \\
0.5-2 \text { kg; repetitions } 10 \times 3 \text {; } \\
\text { Double or single arm rowing with elastic bands: } \\
\text { green or yellow or red; repetitions, } 10 \times 3 \text {; } \\
\text { Standup from a chair or double calf raises: } 10 \times 3 \\
\text { repetitions } \\
\text { Progression: Increase the load or repetitions } \\
\text { Cooling down: (5 min) } \\
\text { Slow activities (slow walking) and self stretching } \\
\text { of all major muscle groups and relaxed breathing }\end{array}$ \\
\hline
\end{tabular}

\section{Step IV - endpoint measurements}

At the completion of the exercise programme (12 weeks) the principal investigator will make an appointment for each participant in order for the questionnaires (ESS, SBQ, SF36) and measurements taken in study I (Table 1) to be repeated. The equipment will be calibrated as required. The participant will then be given an appointment to have an echocardiogram in the Cardiology Research Laboratory. They will be advised to continue the home based programme for long-term health benefits. Each participant will receive a copy of their results. Where permission has been granted the participant's general practitioner will also receive a copy of the end point measurements.

\section{Outcome measures}

The outcome measures will be collected at two time points, pretest (before the intervention) and posttest (at the completion of 12-week program) (Figure 1).

\section{Primary outcome}

\section{4 h ambulatory blood pressure blood pressure}

This will be defined as the mean difference, in $\mathrm{mmHg}$, between pre and post interventions. $24 \mathrm{~h}$ ambulatory BP will be recorded using an Ultralite $^{\mathrm{TM}}$ 90217A ambulatory BP monitor and 92506 ambulatory BP Report Management System (Spacelab Healthcare, WA, USA). $24 \mathrm{~h}$ ambulatory $\mathrm{BP}$ is the recommended method to measure $\mathrm{BP}$ in resistant hypertension according to the AHA/ACC (2017) and ESH/ESC (2018) and it has been commonly used for research. ${ }^{4,11,18,38}$ Office/Clinic BP will also be measured according to the AHA/ACC (2017) and ESH/ESC (2018), guidelines using an Omron digital BP monitor (HEM 7322, Netherlands). ${ }^{4,11}$

\section{Secondary outcomes}

\section{Level of physical activity and sleep fragmentation}

The level of physical activity and sleep parameters (Table 1) will be captured by the ActiGraph GT3XPB, activitrax 
monitor (Pensacola, Florida, USA) in accordance with the instructions in the user manuals. The device, an activity monitor, has previously been used and shown to be a valid and reliable alternative to polysomnography to monitor sleep as well as activity. ${ }^{39}$

\section{Echo cardiac parameters}

The echo cardiac parameters will be defined by the cardiac ultrasound in the facility based in the Dunedin Hospital. The echocardiography will be carried out by technicians under supervision of the interventional cardiologist in the team $(\mathrm{GW})$.

\section{Cardiorespiratory fitness}

The six minute walk test (6MWT) is a valid and reliable measure of cardiorespiratory fitness. ${ }^{40}$ This test is a pragmatic walking test of functional exercise capacity, which can be carried out with minimal equipment. The test will be carried out according to the published guidelines. ${ }^{41}$

\section{Quality of life}

The short form 36 (SF36) which is widely used in assessing quality of life will be administered with the calculation of mean changes in the total score before and after the intervention to determine the quality of life. ${ }^{42}$

\section{Anthropometrics}

Anthropometric data collection (Table 1) will follow the protocols documented in the ESSA Manual. ${ }^{43}$ Body weight and height will be measured using SECA scale (Seca 876) and SECA stadiometer (Seca 217) respectively and body mass will be calculated (weight $[\mathrm{kg}] /$ height $\left.^{2}\left[\mathrm{~m}^{2}\right]\right)$. Hip, waist and neck circumferences will be measured using a standard anthropometric measuring tape (Seca 201).

\section{Data collection and monitoring}

The principal investigator for the project (SD) will collect the data. Data will be collected in person and sufficient time for those participants who have low literacy will be provided to complete the self-reported questionnaires. The primary supervisor (MS) will oversee the data collection as required. All research team members have current CPR certification. If any adverse event occurs during the data collection appropriate first aid measures will be undertaken and the incident will be reported by the primary supervisor to the relevant authority through the standard reporting channels.

The $24 \mathrm{~h}$ ambulatory BP, activity and sleep data will be downloaded to the relevant software programs from the devices. The measurements and data collected by questionnaires will be initially completed electronically via Qualtrics software. The electronic data will be saved on a password-protected computer dedicated to the research, while the hard copies of all data will also be kept under lock and key in the primary supervisor's research office. A backup computer file will be maintained on a separate password protected computer. Only the investigators (four in number) will be able to access the data and no third party will be involved in collection, entering or the processing data. In accordance with the requirement, data will be kept for 10 years and after that it will be discarded according to the standard procedures. The exercise intervention will be carried out by a physiotherapist employed for that purpose, who is outside of the research team, and blinded to the status of the collected data for the participants in the group.

The participants can withdraw from the study any time with no disadvantage to their health care. However, the data obtained up to the point of withdrawal will be used in the study, as per detail in the participant information sheet and consent form. Each participant will be allocated a random ID number generated using Microsoft Excel, by the principal investigator, and no identifiable information relating to participants will be used in the study. Thus, throughout the study and in the publications, or in any other material open to public, no individual data relating to a participant will be identified.

\section{Safety considerations risk and benefits to participants}

This study can be categorized as a low-risk intervention study, and the study population is not vulnerable, based on the guidelines of the Health and Disability Ethics Committees (HDEC), Ministry of Health New Zealand. No invasive clinical or non-clinical procedures or equipment (Table 1) are involved in the proposed study. All measurement (Table 1) and exercise (tread mill, static bicycle, rowing machine, exercise steps, exercise balls, free weights etc.) equipment is categorised as low risk (Class I) according to Therapeutic Goods Administration (TGA) guidelines (2011). The measurement procedures have no or minimal risk - usual muscle soreness/joint pain the day after the exercise programme or falling or tripping whilst walking are potential risks. The ambulatory blood pressure measurements will be recorded during the person's normal daily activity; the exercise programme, questionnaires and the walking test will be carried out at the School of Physiotherapy. The echocardiogram will be repeated at the Dunedin School of Medicine Cardiology Research Laboratory. Thus, there is no significant risk involved with the locations, procedures or measurements to be made.

The exercise programme will be pre-designed, based on a pre-validated programme with aerobic warming up, resistant training, aerobic training and cooling down exercises. Basic gym equipment (treadmill, static bicycle, rowing machine, exercise steps, exercise balls, free weights etc.) will be used for the exercises. All researchers/exercise programme physiotherapist hold at a minimum a current First Aid certificate, and AED emergency equipment is available in each location. Should an adverse event or accident occur, the participant 
will be covered under the Accident Compensation Corporation (ACC) legislation, by ACC. A cardiology consultant is involved in the project as a co-investigator, and is able to advise on procedures.

By participating in the study, the participants will be able to receive a report ( $24 \mathrm{~h}$ ambulatory BP, echocardiogram, risk of obstructive sleep apnea (ESS/SBQ score), physical activity level, anthropometry such as BMI, hip to waist ratio) on their cardiovascular health at the completion of the study. Further, exercise as part of lifestyle modification is recommended to manage $\mathrm{BP}$ in hypertension/resistant hypertension. ${ }^{7}$ Therefore, it is anticipated that the exercise program will have a positive effect on the blood pressure and cardiovascular health of the participants.

\section{Statistical considerations and data analysis}

Mean differences in change of $24 \mathrm{~h}$ ambulatory BP, and office or clinic BP within the three months period will be used to determine the program's effectiveness for the cohort with true resistant hypertension and high risk of OSA. If the sample size is not achieved, or if researchers are unable to obtain follow-up data of the participants due to a high number of withdrawals, the data will be presented as a case series. The comparison of baseline 24h ambulatory BP, echocardiogram parameters, anthropometry parameters, ESS or SBQ score, cardiorespiratory fitness, and activity and sleep levels with an endpoint (after 12-week exercise program) will be undertaken using paired sample t-tests and the $p$-value will be set at $\mathrm{p}<0.05$. The statistical package for social sciences (SPSS) version 25.0 or above for Windows (IBM SPSS version 25.0 or above), New York, USA will be used for the data analysis. ${ }^{15}$ We will adhere to the Consolidated Reporting of Standard Trials (CONSORT) and the CONSORT flow diagram will be used to show the flow of participants, loss of follow-ups and reasons for exclusions etc. ${ }^{44}$

\section{Ethical approval and trial registration}

The current study will conform fully with the principles of the 'Declaration of Helsinki', Good Clinical Practice (GCP) and within the laws and regulations of New Zealand. The study has been registered on the Australian New Zealand Clinical Trials Registry (ANZCTRACTRN12618001881224p), and approved by the Health and Disability Ethics Committee (HDEC), New Zealand (Ethics Ref: 18/CEN/257).

\section{Dissemination of findings}

The key output of the project will be the effectiveness of the exercise as a non pharmacological intervention, to lower blood pressure resistant to medication to a clinically significant level. The use of exercise in managing hypertension is in line with the most recent worldwide and New Zealand, Ministry of Health guidelines but its effectiveness on adults with resistant hypertension and high risk of OSA to date, is unclear. Further, the potential outcome is in line with the mission of the National Heart Foundation of New Zealand, to reduce the premature deaths caused by cardio vascular diseases. ${ }^{2,4,11}$ Results of the study along with results of a narrative review and a systematic review by the same authors, will be published in the peer review journals and presented at conferences.

\section{DISCUSSION}

This pragmatic clinical trial aims to determine effectiveness of physical activity and exercise, a recommended non pharmacological intervention, on a special cohort of people with true resistant hypertension and high risk of OSA. The results of the study have a potential to translate the research evidence surrounding the effectiveness of physical activity and exercise for resistant hypertension into clinical practice. To the best of our knowledge this is the first research of its kind in New Zealand.

The proposed study is significant in several aspects. First, the investigators will adhere to the new guideline of the ACC/AHA 2017 and Ministry of Health, New Zealand 2018 for hypertension (130-139/80-89 mm Hg).,4 Participants will include only those with true resistant hypertension, diagnosed using $24 \mathrm{~h}$ ambulatory $\mathrm{BP}$, the method used to diagnose true resistant hypertension, that has the highest level of recommendation. ${ }^{4,11,18,38}$ Further, this project will likely be the first of its kind, to consider outcomes under the latest guidelines accepted by Ministry of Health, New Zealand, for hypertension, thus creating a new knowledge dimension. ${ }^{2}$ Second, the particular effects of physical exercise in the cohort with true resistant hypertension and high risk of OSA, which is the target group in the study, has not been adequately investigated previously, because studies have not identified those with true resistant hypertension.

As increased physical activity in Stage 1 of hypertension management is the accepted approach for physically inactive people according to the new guideline 2017, which was accepted by New Zealand in 2018., ${ }^{2,5}$ The premise for the study is that increasing physical activity levels will be helpful to reduce BP and to reduce the risk of OSA simultaneously, and thereby to reduce the CVD risk, associated with mortality and morbidity. It is estimated that a reduction in resting blood pressure of at least in $7 \mathrm{mmHg}$ will reduce the CVD risk between $10 \%$ $20 \% .^{21}$ Third, the structural and functional features of the heart of the special subset with true resistant hypertension and high risk of OSA - which will be described for each person from the results of the echocardiogram - will be compared to baseline. This will be a novel feature of the study. Fourth, therapeutic strategies such as exercise will be helpful for improving $\mathrm{BP}$ in the group with true resistant hypertension and high risk of OSA. It should be noted that the predominant recommendation for the management of OSA currently is to control the 
obstruction, which may have little effect on reducing the risk of hypertension. Lastly, the findings have potential to change the direction of research in the area filling in the current knowledge gaps on the topic of true resistant hypertension and OSA rather than adding to the debate. Outcomes may also support the development of modified guidelines for physicians and physiotherapists in managing the cohort with true resistant hypertension and high risk of OSA.

\section{Limitations}

We have identified a few limitations in the proposed study. First, this study will be a non-randomised study and there will be no comparison with a control group. Second, the study population will include Dunedin based adults - Dunedin has a relatively a small population confined to a small geographical area. Third, the study participants' risk of OSA will be determined based on the questionnaires and Activitrax monitoring, not by using polysomnography, the most highly recommended diagnostic method. However, participants whose risk of OSA is high will be referred to the local Sleep Clinic at the conclusion of the study. Lastly the supervised exercise programme will be conducted once a week and the rest of the programme will be an unsupervised home based programme.

\section{Funding: No funding sources}

Conflict of interest: None declared

Ethical approval: The study was approved by the Health and Disability Ethics Committee (HDEC), Ministry of Health, New Zealand (Ethics Ref: 18/CEN/257)

\section{REFERENCES}

1. Smith SM. Epidemiology, prognosis, and treatment of resistant hypertension. Pharmacotherapy. 2013;33(10):1071-86.

2. Ministry of Health New Zealand. Cardiovascular Disease Risk Assessment and Management for Primary Care. Wellington: Ministry of Health: Ministry of Health New Zealand, 2018 14.02.2018. Report No.: HP 6747 Contract No.: HP 6747.

3. McLean RM, Williams S, Mann JI, Miller JC, Parnell WR. Blood pressure and hypertension in New Zealand: results from the 2008/09 Adult Nutrition Survey. NZ Med J. 2013;126(1372):66-79.

4. Whelton PK, Carey RM, Aronow WS, Casey DE Jr, Collins KJ, Himmelfarb CD, et al. 2017 Guideline for the Prevention, Detection, Evaluation, and Management of High Blood Pressure in Adults: Executive Summary: A Report of the American College of Cardiology/American Heart Association Task Force on Clinical Practice Guidelines. J Am Coll Cardiol. 2018;71(19):2199-269.

5. Muntner P, Carey RM, Gidding S, Jones DW, Taler SJ, Wright JT Jr, et al. Potential U.S. Population
Impact of the 2017 ACC/AHA High Blood Pressure Guideline. J Am Coll Cardiol. 2018;71(2):109-18.

6. Sosner P, Guiraud T, Gremeaux V, Arvisais D, Herpin D, Bosquet L. The ambulatory hypotensive effect of aerobic training: a reappraisal through a meta-analysis of selected moderators. Scandinavian J Med Sci Sports. 2017;27(3):327-41.

7. Mancia G, Fagard R, Narkiewicz K, Redon J, Zanchetti A, Bohm M, et al. 2013 ESH/ESC guidelines for the management of arterial hypertension: the Task Force for the Management of Arterial Hypertension of the European Society of Hypertension (ESH) and of the European Society of Cardiology (ESC). Euro Heart J. 2013;34(28):2159219.

8. Cornelissen VA, Smart NA. Exercise training for blood pressure: a systematic review and metaanalysis. J Am Heart Assoc. 2013;2(1):e004473.

9. Chobanian AV, Bakris GL, Black HR, Cuhshman WC, Green LA, Izzo JL, et al. The Seventh Report of the Joint National Committee on Prevention, Detection, Evaluation, and Treatment of High Blood Pressure. The JNC 7 Report. JAMA. 2003;289(19):2560-71.

10. National Institute of Care and Excelence. Hypertension in adults :diagnosis-and-management (NG136). In: National Institute of Care and Excelence, editor. UK: NICE; 2019.

11. Williams B, Mancia G, Spiering W, Agabiti Rosei E, Azizi M, Burnier M, et al. 2018 ESC/ESH Guidelines for the management of arterial hypertension. Europ Heart J. 2018;39(33):3021-104.

12. Calhoun DA, Jones D, Textor S, Goff DC, Murphy $\mathrm{TP}$, Toto RD, et al. Resistant hypertension: diagnosis, evaluation, and treatment: a scientific statement from the American Heart Association Professional Education Committee of the Council for High Blood Pressure Research. Circulation. 2008;117(25):510-26.

13. Carey RM, Calhoun DA, Bakris GL, Brook RD, Daugherty SL, Dennison-Himmelfarb CR, et al. Resistant Hypertension: Detection, Evaluation, and Management: A Scientific Statement From the American Heart Association. Hypertension. 2018;72(5):53-90.

14. Noubiap JJ, Nansseu JR, Nyaga UF, Sime PS, Francis I, Bigna JJ. Global prevalence of resistant hypertension: a meta-analysis of data from 3.2 million patients. Heart. 2019;105(2):98-105.

15. Santos LP, Moraes RS, Vieira PJC, Ash GI, Waclawovsky G, Pescatello LS, et al. Effects of aerobic exercise intensity on ambulatory blood pressure and vascular responses in resistant hypertension: a crossover trial. J Hypertension. 2016;34(7):1317-24.

16. Calhoun DA. Refractory and Resistant Hypertension: Antihypertensive Treatment Failure versus Treatment Resistance. Korean Circulation J. 2016;46(5):593-600. 
17. Doroszko A, Janus A, Szahidewicz-Krupska E, Mazur G, Derkacz A. Resistant Hypertension. Adv Clin Exp Med. 2016;25(1):173-83.

18. Dimeo F, Pagonas N, Seibert F, Arndt R, Zidek W, Westhoff TH. Aerobic Exercise Reduces Blood Pressure in Resistant Hypertension. Hypertension. 2012;60(3):653-8.

19. Sosner P, Gayda M, Dupuy O, Garzon M, Lemasson C, Gremeaux V, et al. Ambulatory blood pressure reduction following high-intensity interval exercise performed in water or dryland condition. J Am Society Hypertens. 2016;10(5):420-8.

20. Brook RD, Appel LJ, Rubenfire M, Ogedegbe G, Bisognano JD, Elliott WJ, et al. Beyond medications and diet: alternative approaches to lowering blood pressure: a scientific statement from the american heart association. Hypertension. 2013;61(6):136083.

21. Burkard T, Mayr M, Winterhalder C, Leonardi L, Eckstein J, Vischer AS. Reliability of single office blood pressure measurements. Heart. 2018;104(14):1173-9.

22. Priou $P$, Le Vaillant $M$, Meslier N, Paris A, Pigeanne T, Nguyen XL, et al. Cumulative Association of Obstructive Sleep Apnea Severity and Short Sleep Duration with the Risk for Hypertension. Plos One. 2014;9(12).

23. Wang AYM. Sleep-Disordered Breathing and Resistant Hypertension. Seminars Nephrol. 2014;34(5):520-31.

24. Pedrosa RP, Drager LF, Gonzaga CC, Sousa MG, de Paula LK, Amaro AC, et al. Obstructive sleep apnea: the most common secondary cause of hypertension associated with resistant hypertension. Hypertension. 2011;58(5):811-7.

25. Cai AP, Wang L, Zhou YL. Hypertension and obstructive sleep apnea. Hypertension Research. 2016;39(6):391-5.

26. de Souza F, Muxfeldt ES, Margallo V, Cortez AF, Cavalcanti AH, Salles GF. Effects of continuous positive airway pressure treatment on aldosterone excretion in patients with obstructive sleep apnoea and resistant hypertension: a randomized controlled trial. J Hypertens. 2017;35(4):837-44.

27. Yu J, Zhou Z, McEvoy D, Anderson CS, Rodgers A, Perkovic V, et al. Association of positive airway pressure with cardiovascular events and death in adults with sleep apnea a systematic review and meta-analysis. JAMA. 2017;318(2):156-66.

28. Dobrosielski DA, Papandreou C, Patil SP, SalasSalvado J. Diet and exercise in the management of obstructive sleep apnoea and cardiovascular disease risk. European Respiratory Rev. 2017;26(144):12.

29. Pagonas N, Vlatsas S, Bauer F, Seibert FS, Zidek $\mathrm{W}$, Babel N, et al. Aerobic versus isometric handgrip exercise in hypertension: a randomized controlled trial. J Hypertens. 2017;35(11):2199-206.

30. Jhons MW. Epworth Sleepiness Scale: Murry W Johns; 1990 - 2015. The official website of The
Epworth Sleepiness Scale. Available at: http://epworthsleepinessscale.com/. Accessed on 3 March 2018.

31. Chiu HY, Chen PY, Chuang LP, Chen NH, Tu YK, Hsieh YJ, et al. Diagnostic accuracy of the Berlin questionnaire, STOP-BANG, STOP, and Epworth sleepiness scale in detecting obstructive sleep apnea: A bivariate meta-analysis. Sleep Med Rev. 2017;36:57-70.

32. Norton K, Norton L. Pre Exercise Screening, Guide to the Australian adult pre-exercise screening system. Published jointly by Exercise and Sports Science Australia, Fitness Australia and Sports Medicine Australia, 2011.

33. Nascimento LS, Santos AC, Lucena JMS, Silva LGO, Almeida AEM, Brasileiro-Santos MS. Acute and chronic effects of aerobic exercise on blood pressure in resistant hypertension: study protocol for a randomized controlled trial. Trials. 2017;18:8.

34. Skinner MA. Personal commiunication with best practice advocay centre, New Zealand, 2018.

35. Hoffmann TC, Glasziou PP, Boutron I, Milne R, Perera R, Moher D, et al. Better reporting of interventions: template for intervention description and replication (TIDieR) checklist and guide. BMJ. 2014;348:g1687.

36. Ministry of Health New Zealand. Eating and activity guidelines for New Zealand adults. HP6271, Ministry of Health, PO Box 5013, Wellington 6154, NZ. 2015.

37. Department of Health and Human Services USA, King AC, Powell KE. 2018 Physical Activity Guidelines Advisory Commitee Scientific Report. Washington Department of Health and Human Services USA, 2018.

38. Guimaraes GV, Cruz LGD, Fernandes-Silva MM, Dorea EL, Bocchi EA. Heated water-based exercise training reduces 24-hour ambulatory blood pressure levels in resistant hypertensive patients: A randomized controlled trial (HEx trial). Int $\mathbf{J}$ Cardiol. 2014;172(2):434-41.

39. Quante M, Kaplan ER, Cailler M, Rueschman M, Wang R, Weng J, et al. Actigraphy-based sleep estimation in adolescents and adults: a comparison with polysomnography using two scoring algorithms. Nature Sci Sleep. 2018;10:13-20.

40. Pugnet G, Marjanovic Z, Deligny C, Boussardon K, Benzidia I, Puyade M, et al. Reproducibility and Utility of the 6-minute Walk Test in Systemic Sclerosis. J Rheumatol. 2018;45(9):1273-80.

41. American Thoracic Society. ATS Statement: Guidelines for the Six-Minute Walk Test. Am J Resp Critical Care Med. 2002;166:111-7.

42. Lins L, Carvalho FM. SF-36 total score as a single measure of health-related quality of life: Scoping review. SAGE Open Med. 2016;4:2050312116671725.

43. Coombes J, Skinner T. ESSA's Student Manual for Health Exercise and Sport Assessment. 1st Edition 
ed. Elsevier Australia. ACN 001002357 (a division of Reed International Books Australia Pty Ltd) Tower 1, 475 Victoria Avenue, Chatswood, NSW 2067: Mosby; 2014.

44. Moher D, Hopewell S, Schulz KF, Montori V, Gotzsche PC, Devereaux PJ, et al. CONSORT 2010 explanation and elaboration: updated guidelines for reporting parallel group randomised trials. BMJ. 2010;340:c869.
Cite this article as: Dassanayake $\mathrm{S}$, Wilkins $\mathrm{G}$, Sole $\mathrm{G}$, Skinner M. Effectiveness of a 12-week physical exercise programme on blood pressure in adults with true resistant hypertension and high risk of obstructive sleep apnoea: a study protocol for a pre-post test non randomised clinical trial. Int J Clin Trials 2020;7(2):112-22. 